\title{
Assessment of Cervical Cancer Screening and its Barriers in 18-50 Year Old Women Referring to Asad Abad Comprehensive Health Centers
}

\author{
Mahnaz Sharifi (MSc) ${ }^{1}$, Ziba Mohammadi (MSc) ${ }^{2}$, Zeinab Makvandi (MSc) $)^{3, *}$, Parisa \\ Rostami (BSc) ${ }^{4}$, Ali Moradi $(\mathbf{P h D})^{5}$
}

${ }^{1}$ Department of Public Health, Asadabad Faculty of Medical Sciences, Hamadan University of Medical Sciences, Hamadan, Iran

${ }^{2}$ Department of Nursing, Asadabad Faculty of Medical Sciences, Hamadan University of Medical Sciences, Hamadan, Iran

${ }^{3}$ Department of Health Education and Promotion, Faculty of Health, Isfahan University of Medical Sciences, Isfahan, Iran

${ }^{4}$ Hamil Health Care Network, Kermanshah University of Medical Sciences, Kermanshah, Iran

${ }^{5}$ Asadabad Health Care Network, Asadabad Faculty of Medical Sciences, Hamadan University of Medical Sciences, Hamadan, Iran

* Corresponding Author: Zeinab Makvandi, Department of Health Education and Promotion, Faculty of Health, Isfahan University of Medical Sciences, Isfahan, Iran. Email: zeinabmakvandi4@gmail.com

\begin{tabular}{|c|c|}
\hline \multirow{3}{*}{$\begin{array}{l}\text { Received: } 29 / 08 / 2017 \\
\text { Accepted: } 17 / 10 / 2017\end{array}$} & Abstract \\
\hline & \multirow{13}{*}{$\begin{array}{l}\text { Background and Objective: Cervical cancer is a major health problem in } \\
\text { Iranian women. The aim of this study was to assess the status of cervical } \\
\text { cancer screening and its barriers in women referred to the health centers of } \\
\text { Asad Abad. } \\
\text { Materials and Methods: In this descriptive-analytic study, } 200 \text { married } \\
\text { women aged } 18-50 \text { years old referred to Asad Abad comprehensive health } \\
\text { centers were selected through cluster sampling in } 2016 \text {. Data were } \\
\text { collected using questionnaire. Chi-square test was used to examine the } \\
\text { relationship between qualitative variables. } \\
\text { Results: More than half ( } 54.5 \% \text { ) of the patients reported a history of } \\
\text { performing a Pap smear test. Out of these patients, } 51.37 \% \text { performed only } \\
\text { one test and } 10.09 \% \text {, repeated the test at standard intervals. } 68.8 \% \text { of the } \\
\text { subjects performed the test on the recommendation of a doctor or midwife. } \\
\text { The results of Chi-square test showed that there was a significant } \\
\text { difference between the prevalence of Pap smear and the number of } \\
\text { pregnancies (P=0.004) and prevention (P=0.01). The most important } \\
\text { reasons for denying Pap smear or doing it irregularly were, lack of } \\
\text { knowledge through the necessity of testing, lack of time and fear of } \\
\text { positive test results. } \\
\text { Conclusion: This study showed low female performance in performing Pap } \\
\text { smear and the most important barrier was lack of awareness. Therefore it is } \\
\text { suggested to conduct different educational programs which make women } \\
\text { sensitive about the benefits of screening and early diagnosis of disease in } \\
\text { early stages. }\end{array}$} \\
\hline & \\
\hline \multirow{12}{*}{$\begin{array}{l}\text { How to Cite this Article: } \\
\text { Sharifi M, Mohammadi Z, } \\
\text { Makvandi Z, Rostami P, Moradi } \\
\text { A. Assessment of cervical } \\
\text { Cancer Screening and its } \\
\text { Barriers in 18-50 Year Old } \\
\text { Women Referring to Asad Abad } \\
\text { Comprehensive Health Centers. } \\
\text { Pajouhan Scientific Journal. } \\
\text { 2018; 16(2): 35-45. DOI: } \\
\text { 10.21859/psj.16.2.35 }\end{array}$} & \\
\hline & \\
\hline & \\
\hline & \\
\hline & \\
\hline & \\
\hline & \\
\hline & \\
\hline & \\
\hline & \\
\hline & \\
\hline & Keywords: Cervical Cancer; Screening; Women \\
\hline
\end{tabular}


dof: $10.21859 /$ psj. 16.2 .35

\section{بررسى وضعيت غربالكرى سرطان دهانه رحم و موانع انجام آن در زنان •ه-11 سال مراجعه كننده به مراكز جامع سلامت شهر اسد آباد}

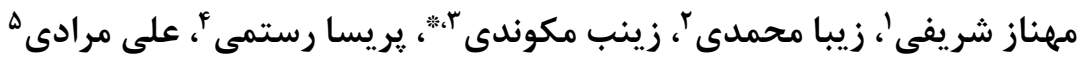

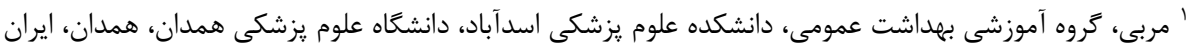

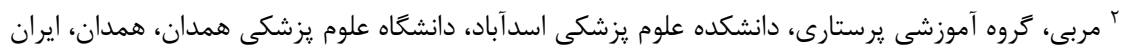

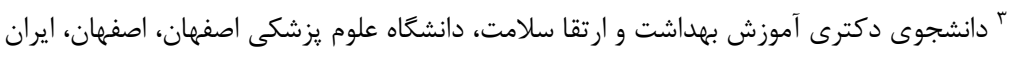

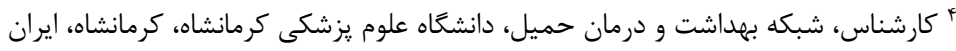

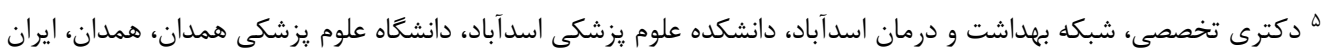

* * نويسنده مسئول: زينب مكوندى، دانشخاه علوم يزشكى اصفهان، اصفهان، ايران. ايميل: zeinabmakvandi@gmail.com

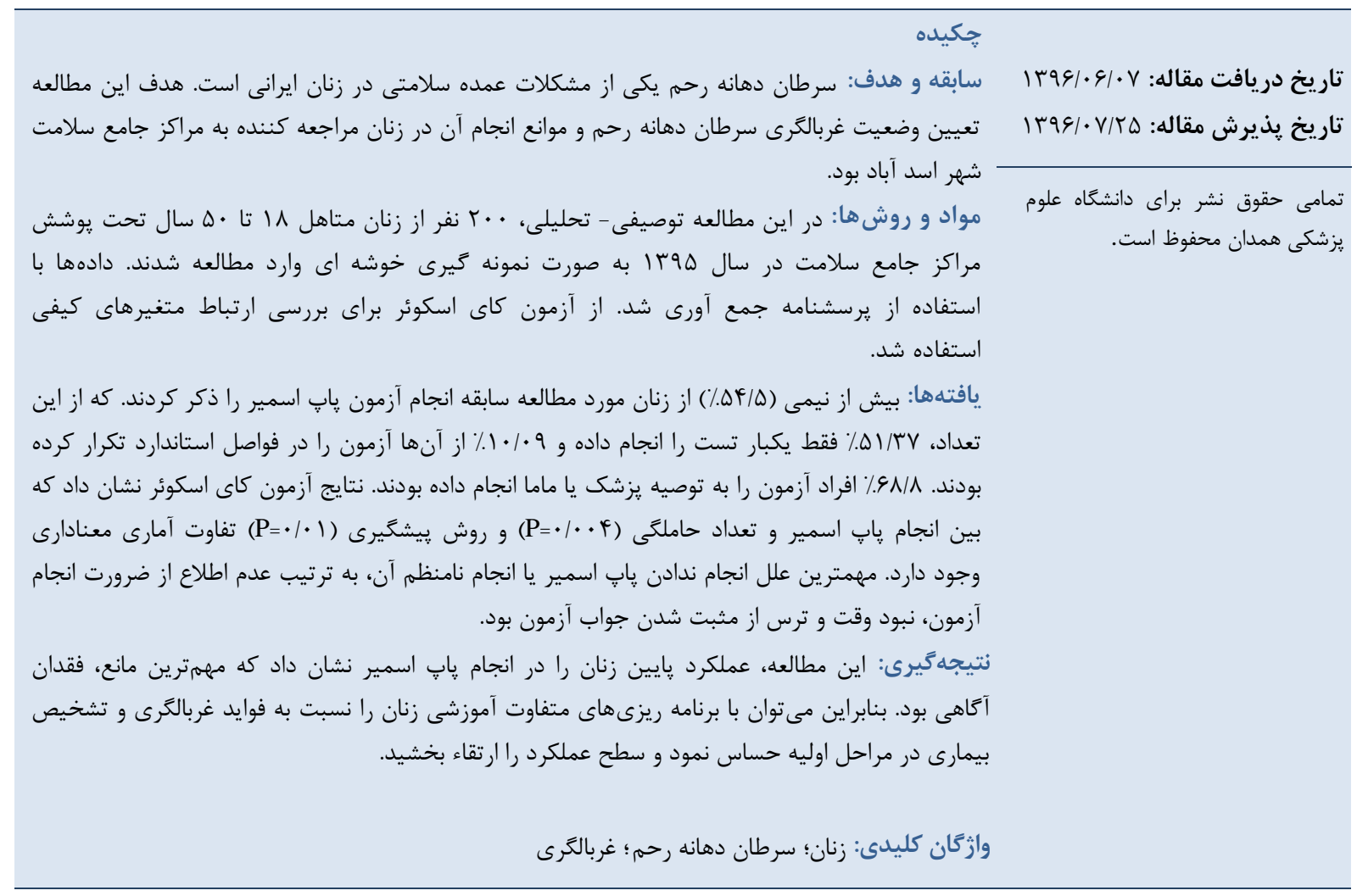

زنان كشورهاى در حال توسعه است. هر سال از هر بآ ازن

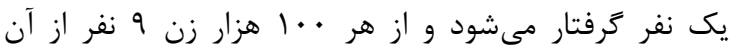

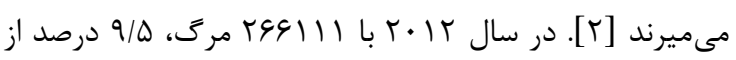

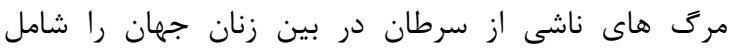

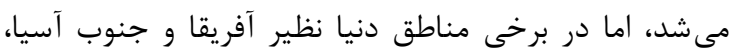

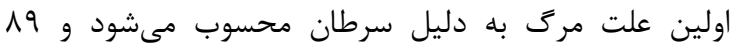

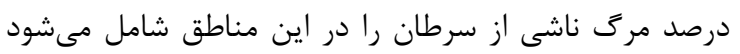

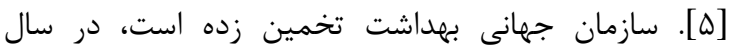

سرطان يكى از سه علت اول مرك و مير در جهان

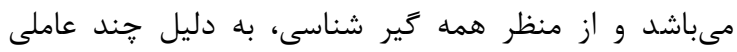

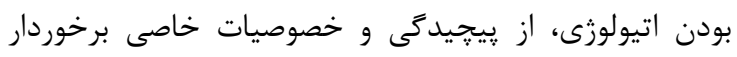

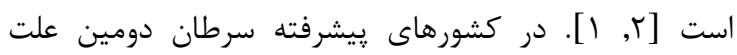

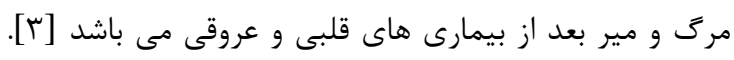

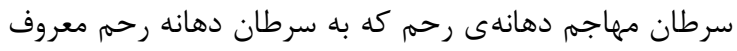

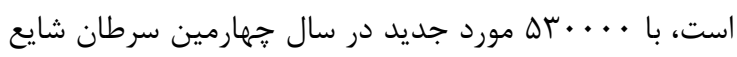

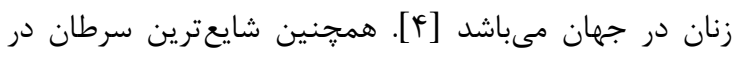


اولين مقاربت، شركاى جنسى متعدد، سيعار كشيدن، مرتبه

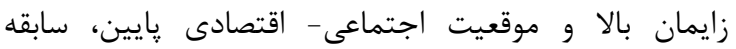

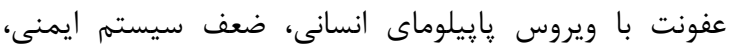
مولتى ياريتى) تعداد باردارى و زايمان زياد (ابتلاء مكرر به به

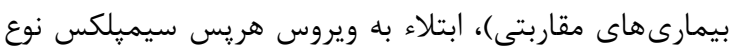
r (قرار گرفتن در معرض دى اتيل بسترول (DES) در دوره

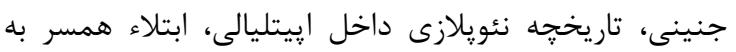

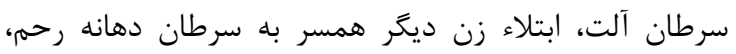

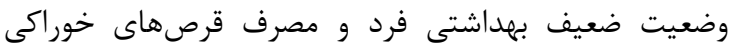

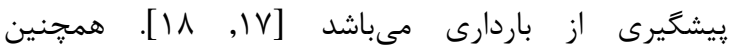
يزوهشهاى به عمل آمده گوياى اين مطلب است كه زبنان آنان

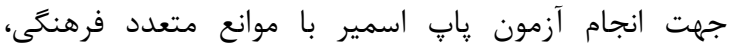

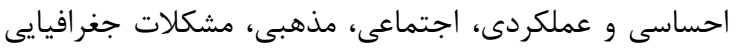

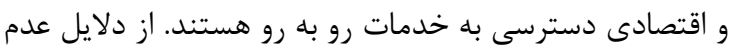

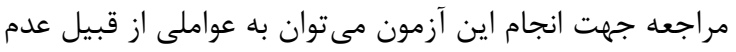

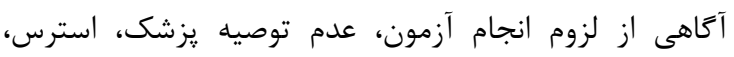

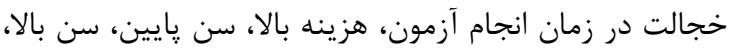

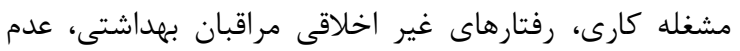

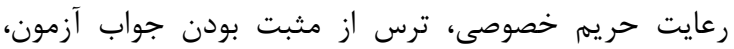

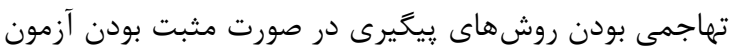

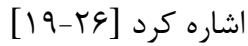

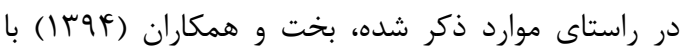

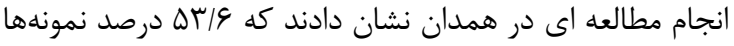

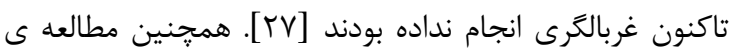

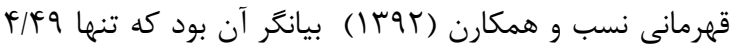
درصد زنان مراجعه كننده به مراكز بهداشتى درمانى شهر تبريز

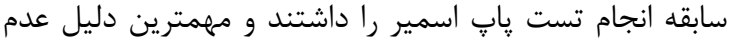

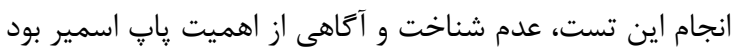

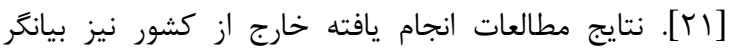

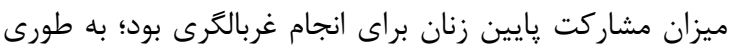

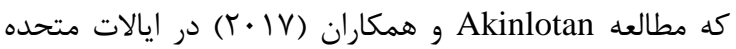

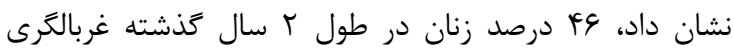

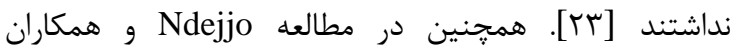

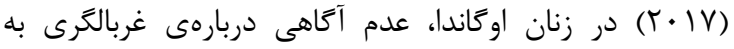

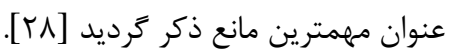
با توجه به آمار مذكور، اهميت موضوع و اين كه كهرديت سرطانها بر روى جنبه هاى مختلف كيفيت زندكى از جمله جنبه بهاى فيزيكى، روانى، اجتماعى و روحى و بطور كلى سلامت و رفاه

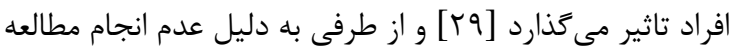

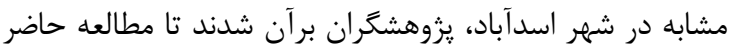

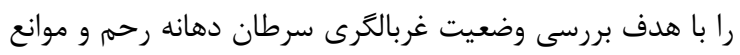

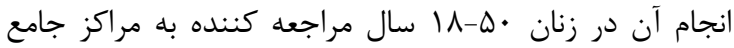

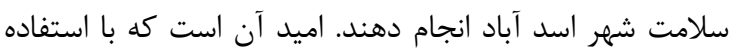
از اطلاعات بدست آمده از اين يزوهش، بتوان كام دهر موثرى براى

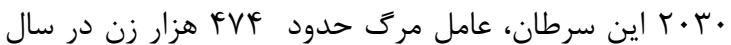

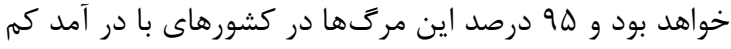

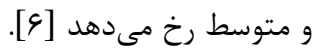

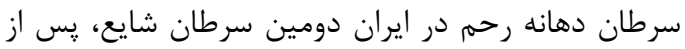

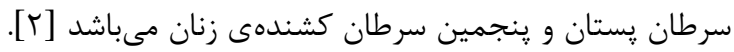

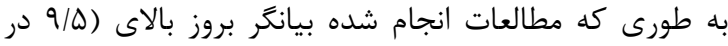

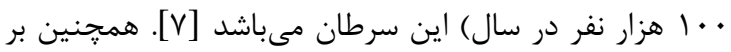

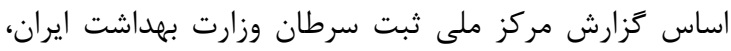

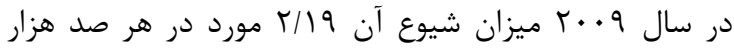

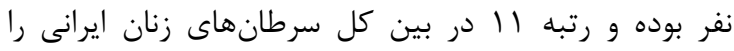

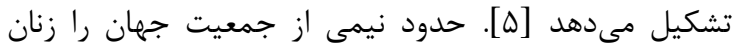

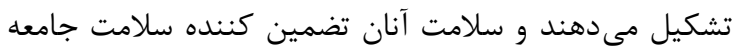
بوده و از اهميت ويزهاى برخوردار است. حفظ سلامت زنان در

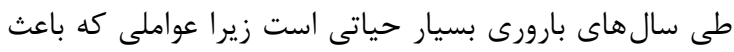

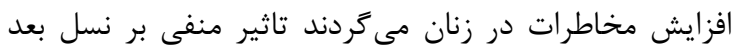

خواهند داشت [1].]

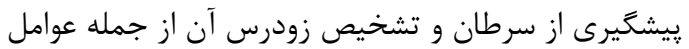

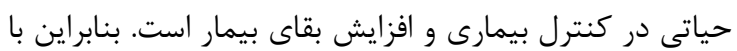

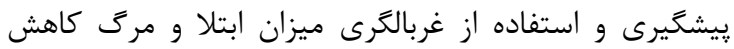

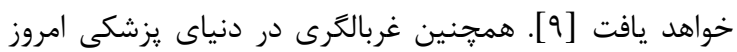

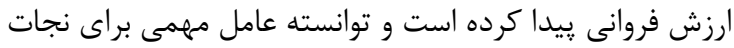

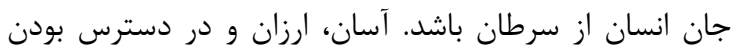

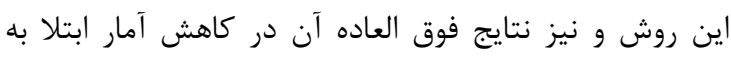

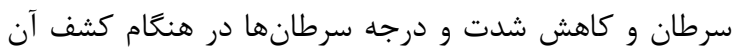

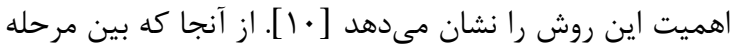

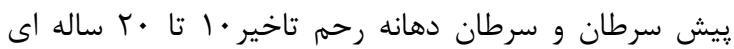

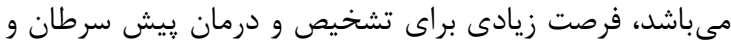

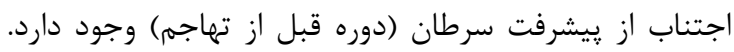

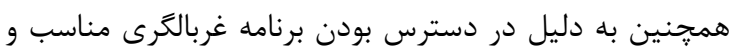

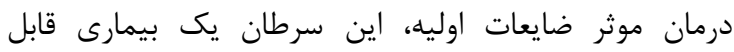

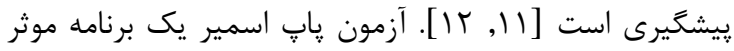

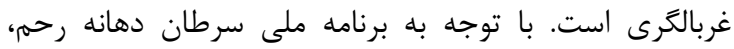

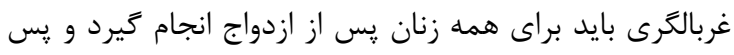

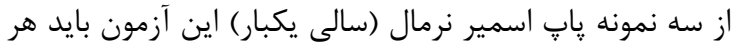

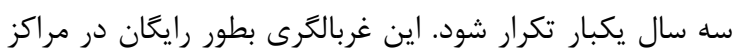

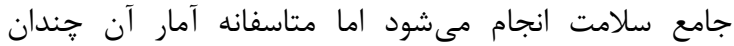

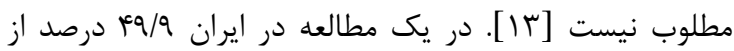

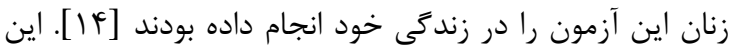

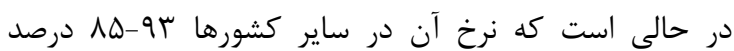

كزارش شده است [هات

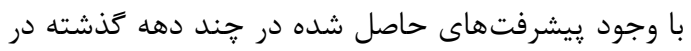

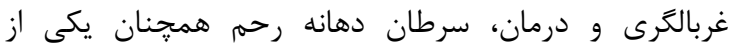

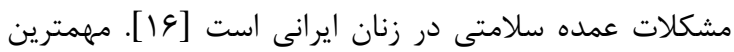

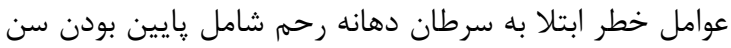


پِإ اسمير تا r سال و در صورت منفى بودن هر سه سال

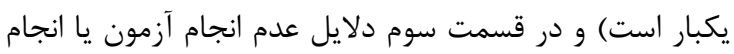

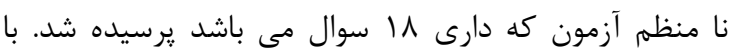

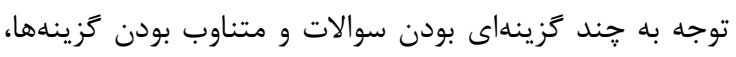

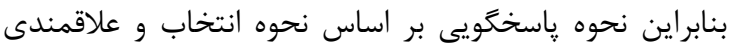

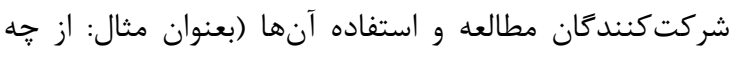

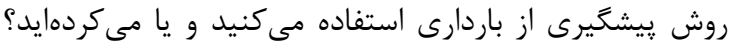

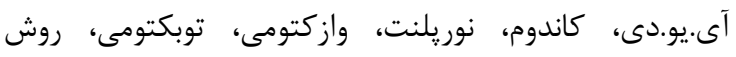

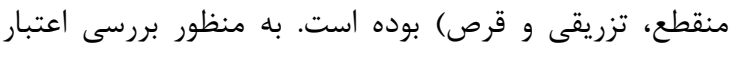

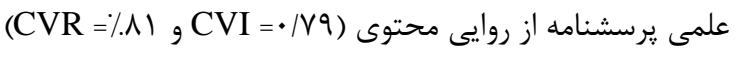

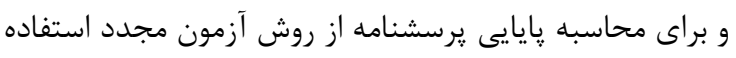

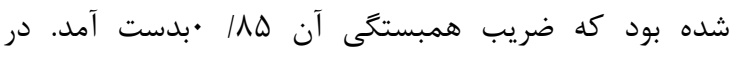

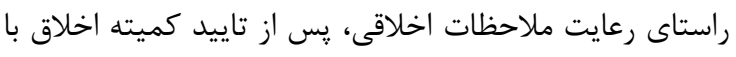

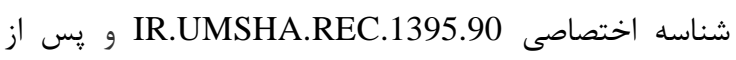

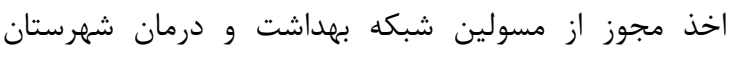

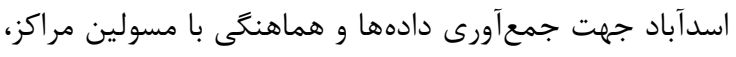

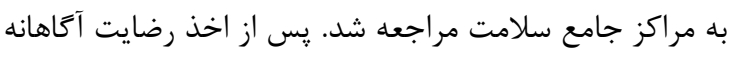

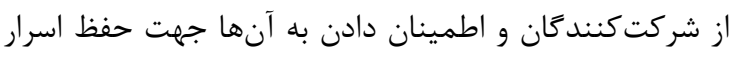

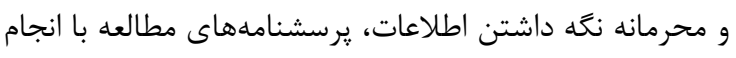

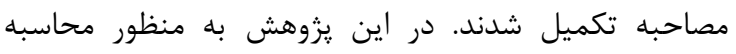

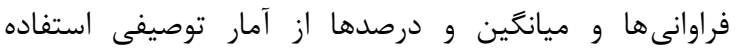

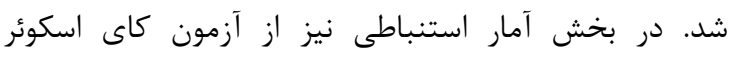

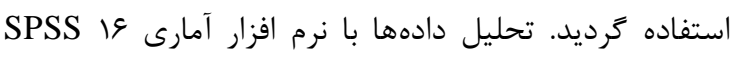

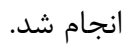

\section{يافتهها}

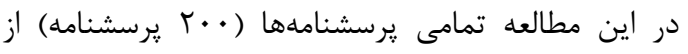

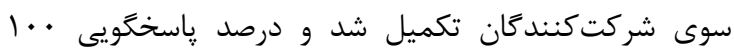

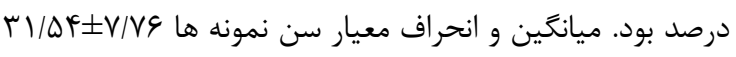

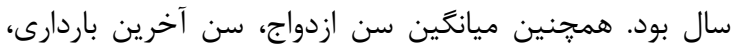

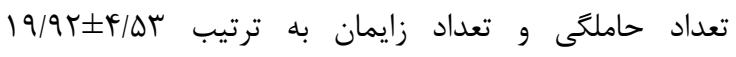
سال،

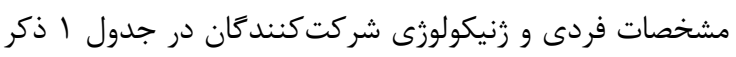

شده است.

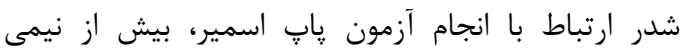

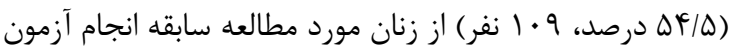

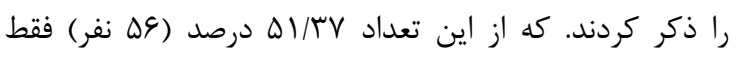

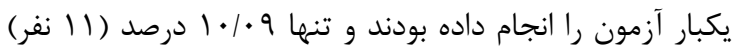
از آنها آزمون را در فواصل استاندارد تكرار كرده بوداد بودند.

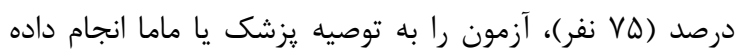

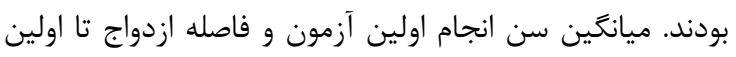

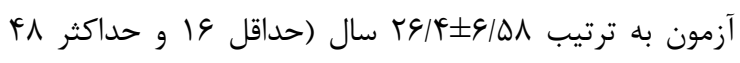

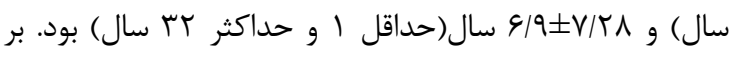

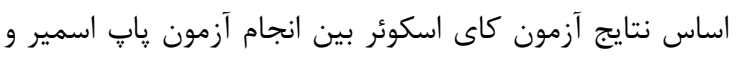

رفع موانع مرتبط با غربالكَى سرطان دهانه رحم در زنان برداشت.

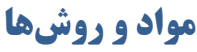

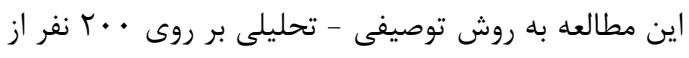

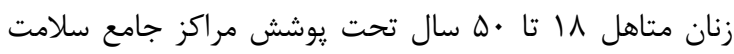

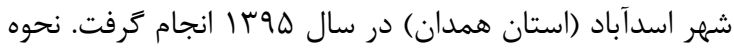

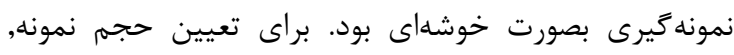

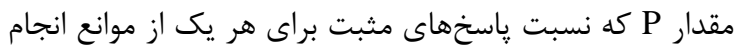

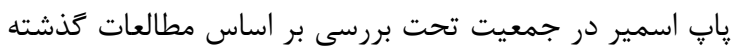

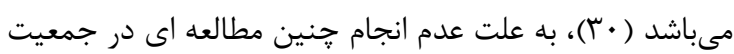

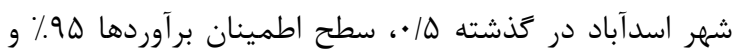

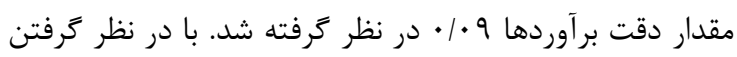

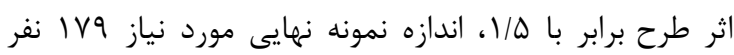

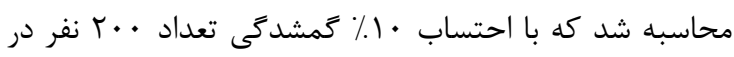

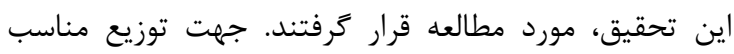

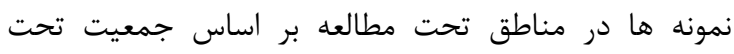

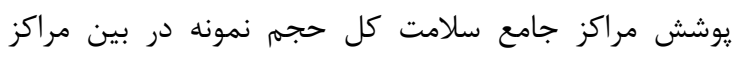

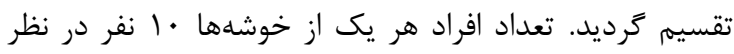

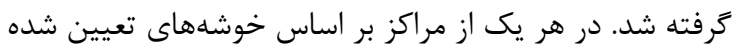

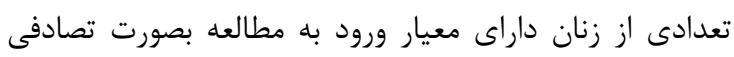

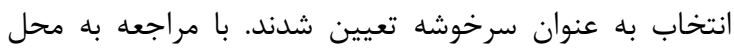

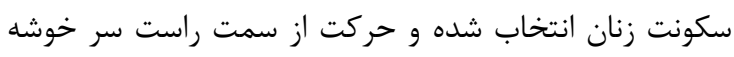

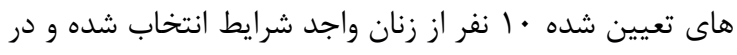

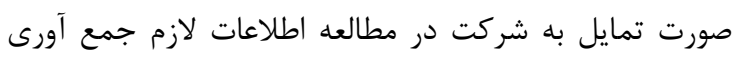

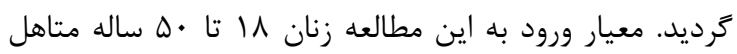

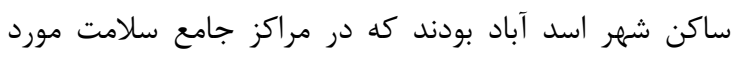

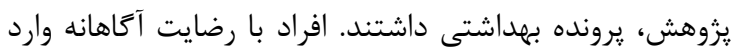

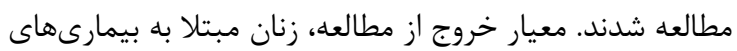
شايع دستخاه تناسلى بود.

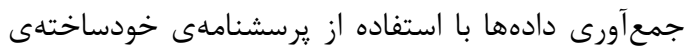

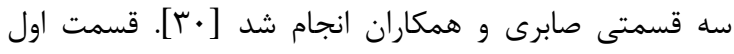

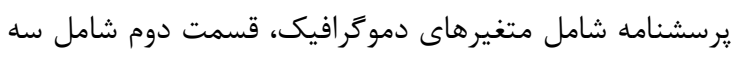

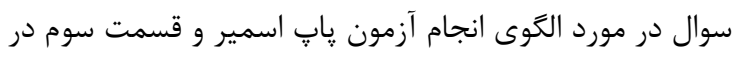

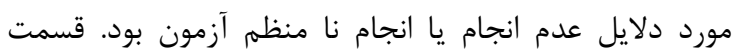

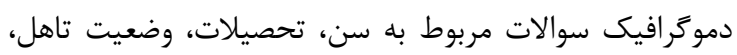

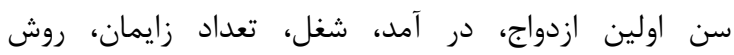

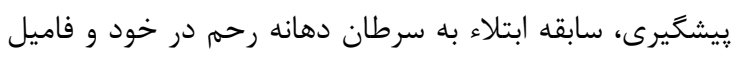

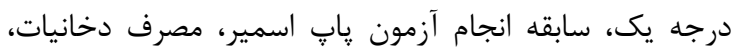

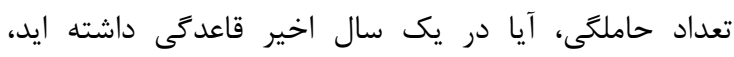

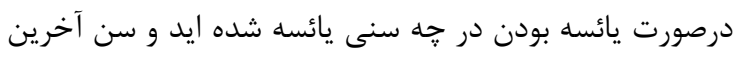

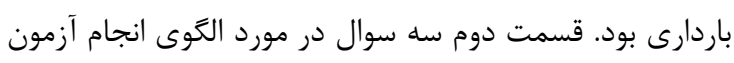

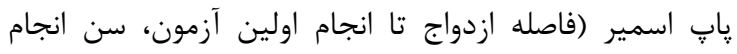
اولين آزمون و رعايت فواصل استاندارد (انجام سالانه آزمون آنام آنام آنامن 
جدول ا: مشخصات فردى و زنيكولوزى زنان شهر اسدآباد

\begin{tabular}{|c|c|c|c|}
\hline تعداد (درصد) & متغير ها & تعداد (درصد) & متغيرها \\
\hline & \multicolumn{2}{|l|}{ مصرف دخانيات } & كروه سنى (سال) \\
\hline$q \Delta(F \vee / Q)$ & 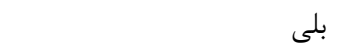 & $\mid \& \&(V K)$ & كمتر و مساوى فـ \\
\hline \multirow[t]{2}{*}{$1 \cdot \Delta(\Delta T / \Delta)$} & 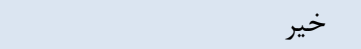 & $\Delta F(T V)$ & 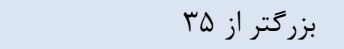 \\
\hline & \multicolumn{2}{|l|}{ سن ازدواج (سال) } & تحصيلات \\
\hline$I r \Delta(\xi \vee / \Delta)$ & كمتر و مساوى 11 & $\mathbb{I r}(9)$ & بى سواد \\
\hline \multirow[t]{2}{*}{$Q D(T Y / \Delta)$} & 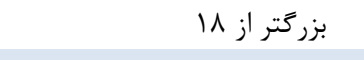 & $F \cdot(r \cdot)$ & ابتدائى \\
\hline & 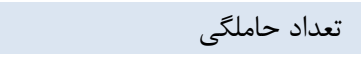 & $\longleftrightarrow \wedge(19)$ & راهنمايى \\
\hline $\mid \Delta r(V \& \mid \Delta)$ & كمتر و مساوى & $\varphi \cdot(r \cdot)$ & دييلم \\
\hline \multirow[t]{2}{*}{$F V(Y M / Q)$} & بزر بتر از r & $\Delta \cdot(T \Delta)$ & دانشخاهى \\
\hline & \multicolumn{2}{|l|}{ 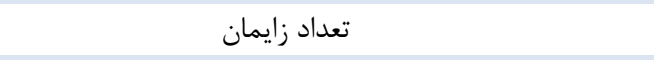 } & وضعيت تأهل \\
\hline $1 \wedge \Delta(9 T / \Delta)$ & كمتر و مساوى & $194(9 \vee)$ & 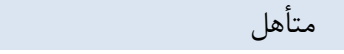 \\
\hline \multirow[t]{2}{*}{$1 \Delta(V / \Delta)$} & 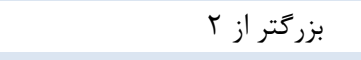 & $r(1 / \Delta)$ & بيوه \\
\hline & سن آخرين باردارى & $r(1 / \Delta)$ & 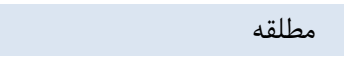 \\
\hline $1 \wedge 9(9 \% / 0)$ & كمتر و مساوى & & شغل \\
\hline \multirow[t]{2}{*}{$11(\Delta / \Delta)$} & 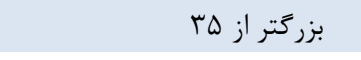 & $19 \cdot(\Lambda \cdot)$ & 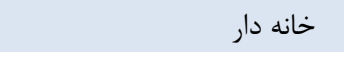 \\
\hline & وجود قاعدىى در يك سال اخير & TY(IT) & 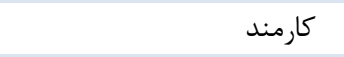 \\
\hline$F q(Y Y / Q)$ & 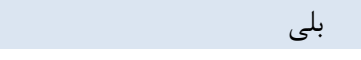 & $\operatorname{Ir}(9)$ & 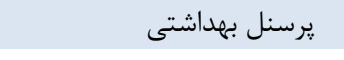 \\
\hline \multirow[t]{2}{*}{$1 \Delta(V / \Delta)$} & 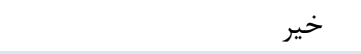 & $f(r)$ & ساير موارد \\
\hline & روش بِيشكيرى از باردارى & & 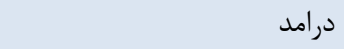 \\
\hline$F q(T F / \Delta)$ & 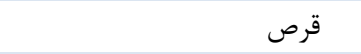 & $111(\Delta 9)$ & ضعيف \\
\hline $1 \Delta(\Lambda)$ & آى.يو.دى & $r V(\mid \wedge / \Delta)$ & 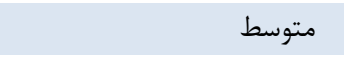 \\
\hline$F \mid(r I)$ & كاندوم & $F \Delta(Y Y / Q)$ & 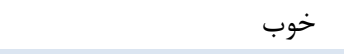 \\
\hline$V F(T V)$ & منقطع & & سابقه خانوادَى ابتلا به سرطان \\
\hline $\operatorname{lf}(V)$ & 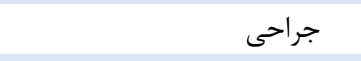 & $\Lambda(\uparrow)$ & 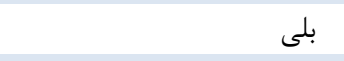 \\
\hline$\Delta(T / \Delta)$ & تزريقى & $194(98)$ & خير \\
\hline
\end{tabular}

يك سال اخير و سابقه ابتلا به سرطان در فاميل رابطه آمارى

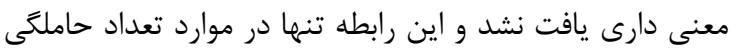

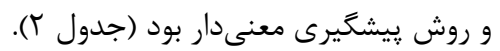

متغيرهاى دموكرافيك و زنيكولوزى، سن، سطح تحصيلات،

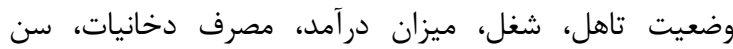

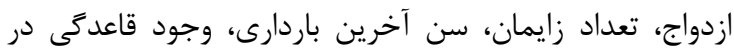

جدول r: ارتباط انجام آزمون پِا اسمير با مشخصات فردى و زنيكولوزى زنان شهر اسدآباد

\begin{tabular}{|c|c|c|c|c|}
\hline \multirow{3}{*}{ P-value } & \multicolumn{2}{|c|}{ آزمون ياب اسمير } & & \multirow{3}{*}{ متغيرها } \\
\hline & خير (n=91) & بله (-9=1.9) & & \\
\hline & تعداد (درصد) & تعداد (درصد) & & \\
\hline \multirow{2}{*}{$\cdot / r \Delta f$} & $V \cdot(V \& / 9 Y)$ & $V \varphi(99 / V T)$ & كمتر و مساوى هـ & \multirow{2}{*}{ حروه سنى (سال) } \\
\hline & $r \mid(Y T / \cdot 1)$ & $r r(r \cdot / r \Lambda)$ & بزرَتر از هـ & \\
\hline \multirow{5}{*}{ - IGAT } & $\varphi(\mathcal{G} / \mathcal{\varepsilon})$ & $\varphi(\Delta / \Delta)$ & بى سواد & \multirow{5}{*}{ تحصيلات } \\
\hline & $r \cdot(r) / 9 V)$ & $r \cdot(\mid N / K Y)$ & ابتدائى & \\
\hline & $19(\mid V / \Delta \Lambda)$ & $r T(r \cdot / 1 \Lambda)$ & راهنمايى & \\
\hline & $r r(T \Delta / T V)$ & $r V(r r / q Y)$ & دييلم & \\
\hline & $r \varphi(T \wedge / \wedge \Delta)$ & $T F(Y Y / \cdot Y)$ & دانشكاهى & \\
\hline \multirow{3}{*}{$\cdot / 4 I V$} & 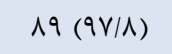 & 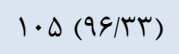 & متأهل & \multirow{3}{*}{ وضعيت تأهل } \\
\hline & $\cdot(\cdot)$ & $\Gamma(T / V \Delta)$ & بيوه & \\
\hline & $(r / T) Y$ & $1(\cdot / 9 r)$ & مطلقه & \\
\hline
\end{tabular}




\begin{tabular}{|c|c|c|c|c|}
\hline & & & & ادامه جدول ا. \\
\hline \multirow{4}{*}{$\cdot / 991$} & $V r(\Lambda \cdot / r I)$ & $\wedge \vee(\vee ৭ / \wedge)$ & خانه دار & \multirow{4}{*}{ شغل } \\
\hline & $11(\mid r / 1)$ & $1 r(11 / 9 T)$ & كارمند & \\
\hline & $\Delta(\Delta / \uparrow q)$ & $V(g / F r)$ & يرسنل بهداشتى & \\
\hline & $r(T / T)$ & $r(1 / \wedge \varepsilon)$ & ساير موارد & \\
\hline \multirow{3}{*}{. ATr } & $\Delta r(\Delta \Lambda / T Y)$ & $\angle \Delta(\Delta Q / Q \Psi)$ & ضعيف & \multirow{3}{*}{ در مرامد } \\
\hline & $r \cdot(r / / 9 V)$ & IV $(\mid \Delta / 9)$ & متوسط & \\
\hline & $11(19 / \vee 9)$ & $Y V(Y F / V Y)$ & خوب & \\
\hline \multirow{2}{*}{ - /VqF } & $\mathcal{F}(\boldsymbol{F} / \mathcal{F})$ & $f(r / 99)$ & 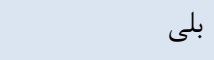 & \multirow{2}{*}{ مصرف دخانيات } \\
\hline & $\wedge \vee(9 \Delta / 9)$ & $1 \cdot \Delta\left(q g / M^{\prime} F\right)$ & خير & \\
\hline \multirow{2}{*}{.$/ I r v$} & $r \wedge(f \backslash / V \Delta)$ & $\Delta V(\Delta T / T q)$ & كمتر و مساوى11| & \multirow{2}{*}{ سن ازدواج (سال) } \\
\hline & $\Delta r(\Delta \Lambda / r \Delta)$ & $\Delta T(F \vee / V I)$ & بزركتر ازي| & \\
\hline \multirow{2}{*}{$* \cdot 1 \cdot r$} & $\vee \backslash(\vee \wedge)$ & $G F(\Delta \Lambda / V I)$ & كمتر و مساوى & \multirow{2}{*}{ تعداد حاملىى } \\
\hline & $r \cdot(T r)$ & $F \Delta(\{\backslash / r q)$ & بزركتر ازr & \\
\hline \multirow{2}{*}{$\cdot / \cdot V 1$} & $V \Delta(\Lambda T / F))$ & $\vee \wedge(V / / \Delta \Delta)$ & كمتر و مساوى & \multirow{2}{*}{ تعداد زايمان } \\
\hline & $19(1 V / 09)$ & $r(T N / F \Delta)$ & بزركتر ازب & \\
\hline \multirow{2}{*}{. MTL } & 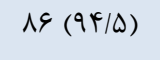 & $99(9 \cdot / \wedge r)$ & كمتر و مساوىهـ & \multirow{2}{*}{ سن آخرين باردارى } \\
\hline & $\Delta(\Delta / \Delta)$ & $1 \cdot(9 / 11)$ & بزركتر ازها & \\
\hline \multirow{2}{*}{. $|\Delta T|$} & $\wedge V(9 \Delta / 9)$ & $1 \cdot r(q r / \Delta V)$ & بله & \multirow{2}{*}{ وجير قاعدىى در يك سال } \\
\hline & $F(F / F)$ & $V(\varepsilon / F T)$ & خير & \\
\hline \multirow{6}{*}{$* \cdot 1 \cdot 1$} & $r V(Y Q / \& V)$ & $r T(r \cdot / / \Lambda)$ & قرص & \multirow{6}{*}{ روش پيشخيرى از باردارى } \\
\hline & $r(\Gamma / \Gamma)$ & $1 T(11)$ & آى.يو.دى & \\
\hline & $r T(T Y / M \Lambda)$ & $19(1 V / F r)$ & كاندوم & \\
\hline & $r \wedge(r \cdot / V \varepsilon)$ & $F \in(F T / T)$ & منقطع & \\
\hline & $\Delta(\varepsilon)$ & $9(9 / 19)$ & جراحى & \\
\hline & $\Delta(\varphi)$ & $\cdot(\cdot)$ & تزريقى & \\
\hline \multirow{2}{*}{.$/ 999$} & $\operatorname{IV}(\mid \Lambda / 9 \wedge)$ & $r \cdot(\mid \Lambda / r Y)$ & بلى بلى & \multirow{2}{*}{ 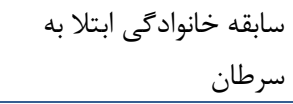 } \\
\hline & $V F(\Lambda I / K T)$ & AV $(\lambda \mid / 94)$ & خير & \\
\hline
\end{tabular}

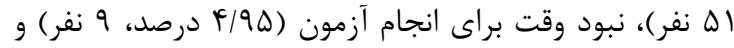

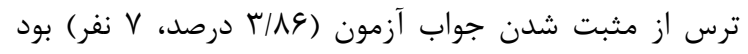

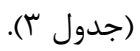

همجنين يافتهاى يزوهش نشان داد كه مهمترين علل

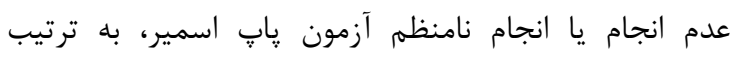

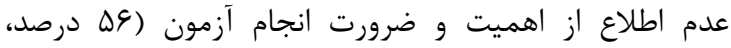

جدول ץ: علل عدم انجام آزمون پاب اسمير به ترتيب اولويت در زنان اسدآباد (1) (n=9)

\begin{tabular}{|c|c|c|}
\hline 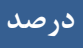 & 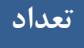 & 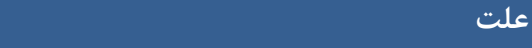 \\
\hline$\Delta \varphi$ & Q1 & راجع به اهميت و ضرورت آن اطلاعى نداشتم. \\
\hline $4 / 90$ & 9 & وقت براى انجام آزمون ندارم. \\
\hline $\mathrm{V} / \mathrm{V}$ & $\checkmark$ & از مثبت بودن جواب آزمون مىترسم. \\
\hline 4/9 & 4 & مى ترسم هنعام انجام آزمون، آسيبى به من برسد. \\
\hline 9/9 & \& & توانايى برداخت هزينه انجام آزمون را ندارم. \\
\hline r/ & r & نسبت به سلامتى خود بى توجه هسته. \\
\hline$r / \mu$ & r & جون فعاليت جنسى ندارم، لازم نيست انجام دهم. \\
\hline$r / T)$ & 1 & از درد هنكًام انجام آزمون مىترسم. \\
\hline$r / \mu l$ & 1 & انجام آزمون برايم اضطراب آور است. \\
\hline$r / \mu l$ & 1 & احساس شرم و خجالت مانع از مراجعه مىشود. \\
\hline$r / T 1$ & 1 & مركزى كه آزمون در آنجا انجام مىشود، دور است. \\
\hline$r / T)$ & 1 & در مكان انجام آزمون احساس راحتى نمى كنم. \\
\hline
\end{tabular}


كنترل، سابقه حداقل يكبار انجام آزمون پِاٍ اسمير را داشتند و

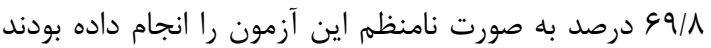

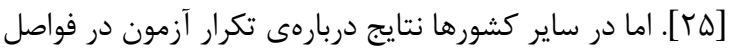

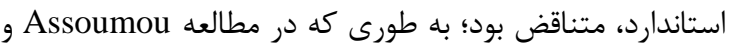

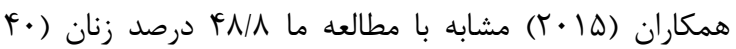

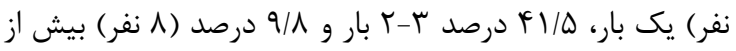

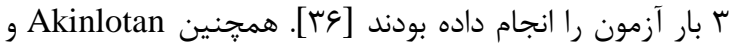

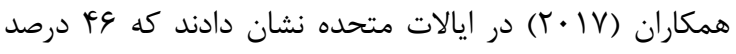

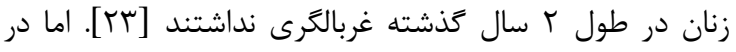
مطالعه ى Chorley و همكاران برخلاف مطالعه ى حنى حاضر، اكثريت زنان حداقل يكبار در غربالكرى شركت كرده بودان بوند

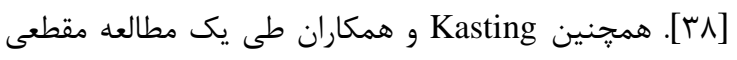

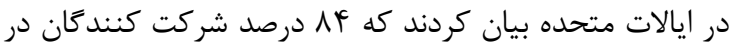

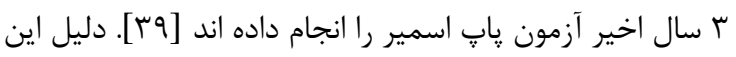

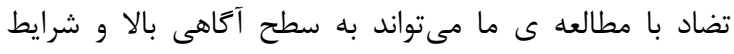

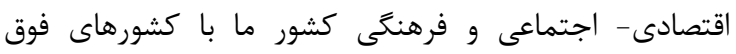
بركردد.

در اين مطالعه ميانگين سن انجام اولين آزمون و فاصله

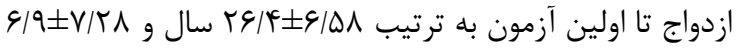

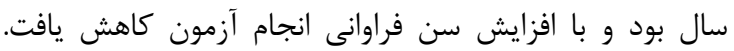

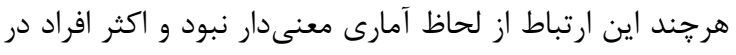

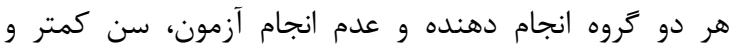
مساوى هץ سال داشتند. در همين رابطه صابرى و همكاران

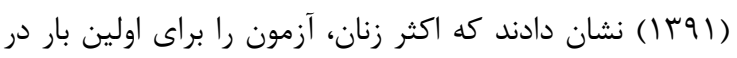

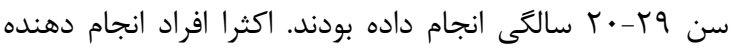

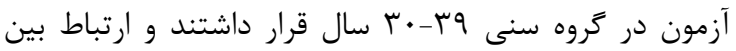
سن و انجام آزمون معنى دار نبود. اما فاصله ازدواج تا اولين

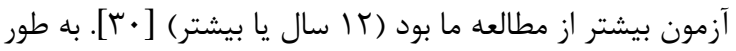

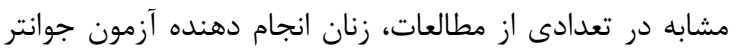

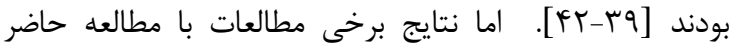

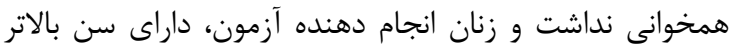

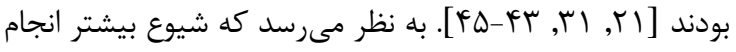
آزمون در زنان جوانتر، ناشى از مراجعه بيشتر آنان به مراكز جامع سلامت جهت دريافت خدمات مادر-كودك باشد.

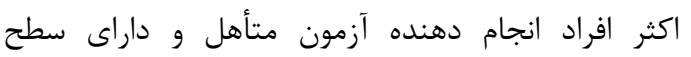

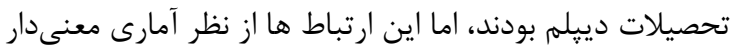

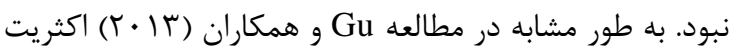
زنانى كه تمايل به انجام غربالكرى در آينده داشتند، متأهل و

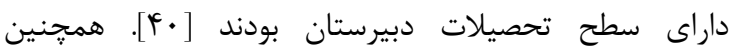

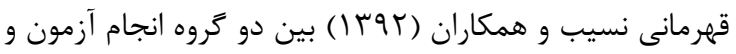

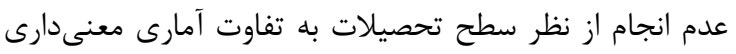

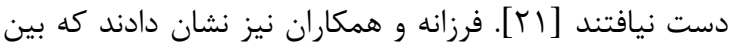

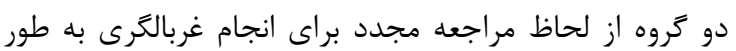

در اين يزوهش وضعيت غربالكرى سرطان دهانه رحم و

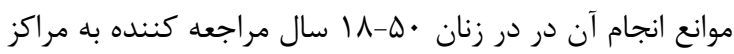

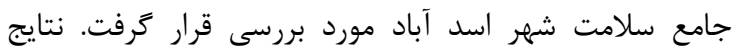

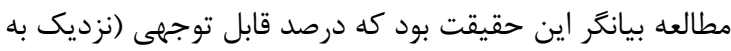

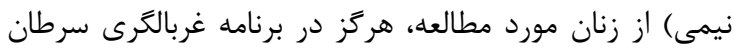

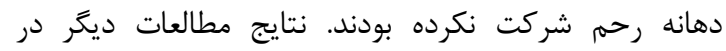

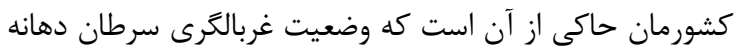

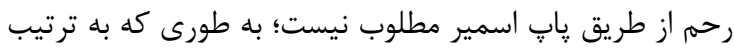
تنها

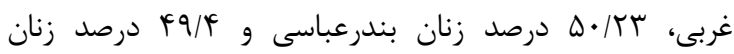

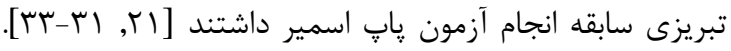

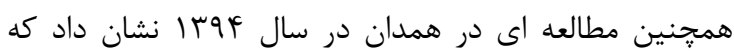

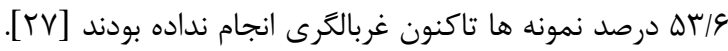

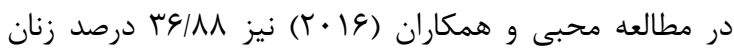

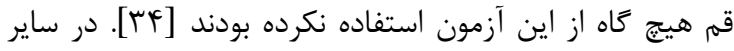

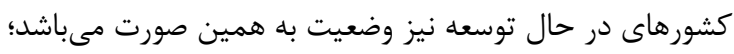

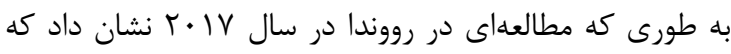

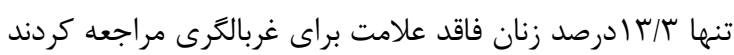

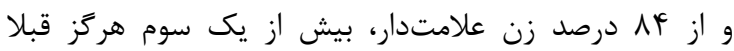

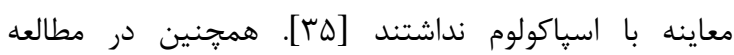
Assoumou

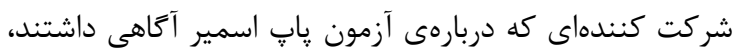

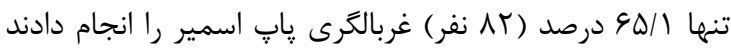

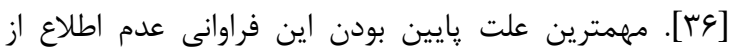
اهميت و ضرورت انجام آزمون بود.

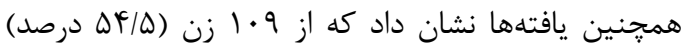

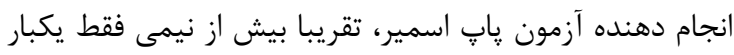

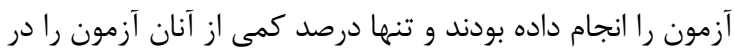

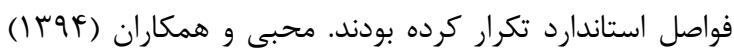

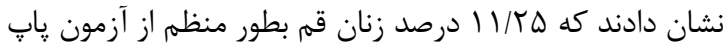

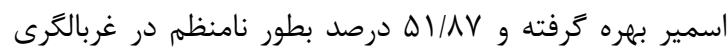

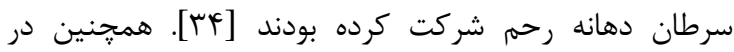

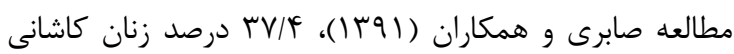

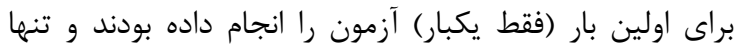

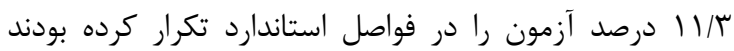

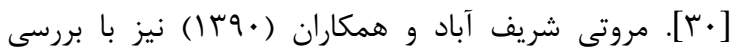

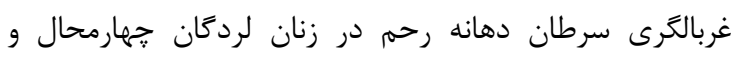

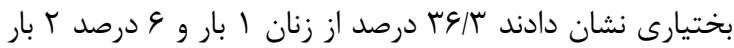

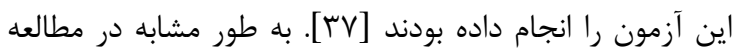

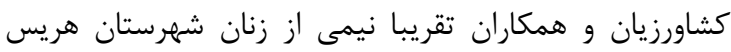
(آذربايجان شرقى) يكبار آزمون را انجام دادند [11]

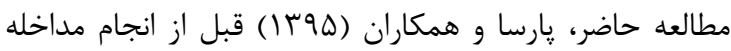

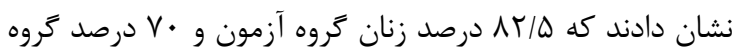


كروه، سابقه خانوادگى سرطان را ذكر نكردند. در همين رابطه

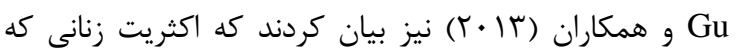

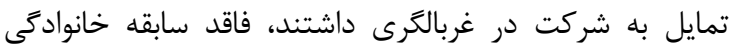

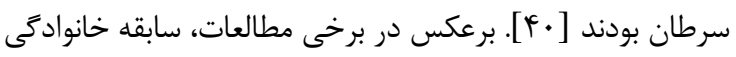

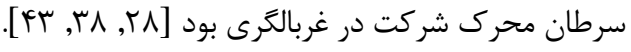

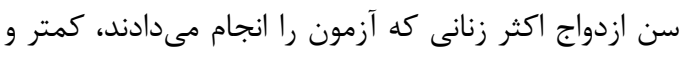

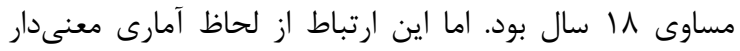

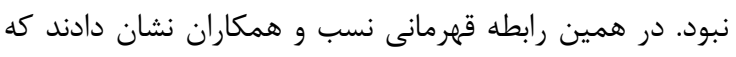

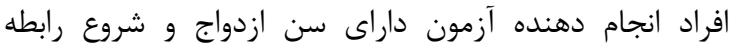

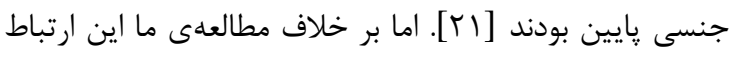

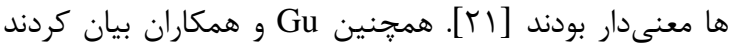

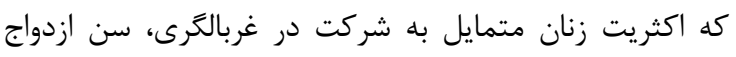

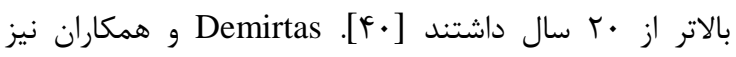

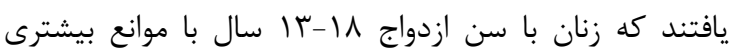

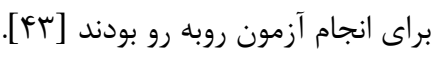

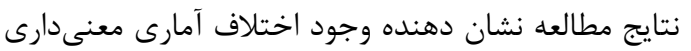

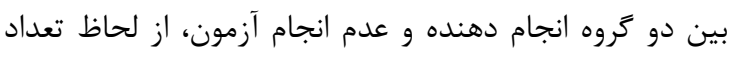

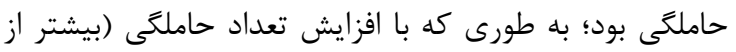

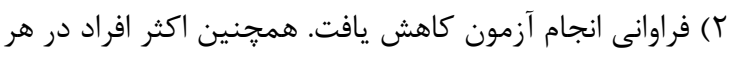

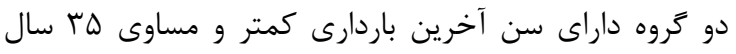

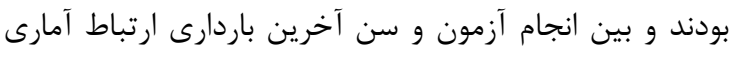

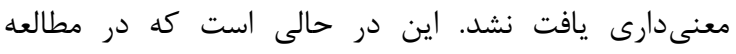
Assoumou

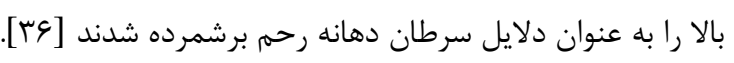

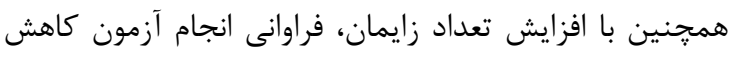

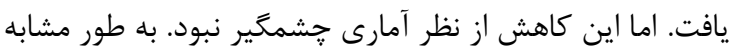

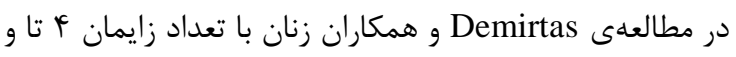

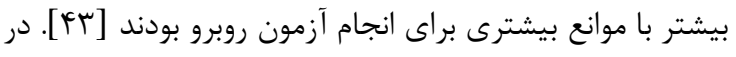

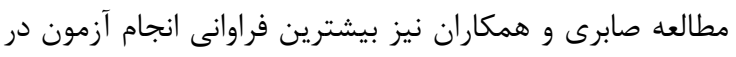

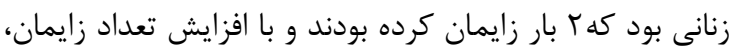

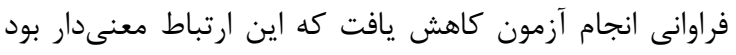

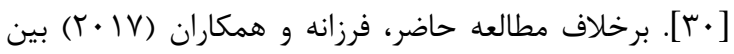

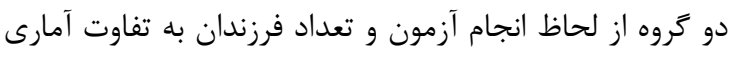

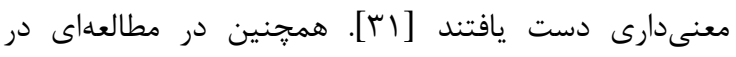

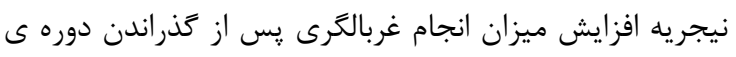

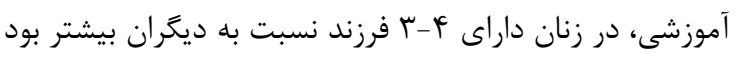

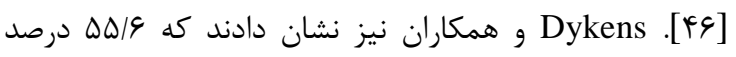

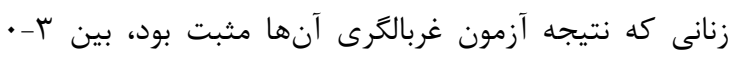

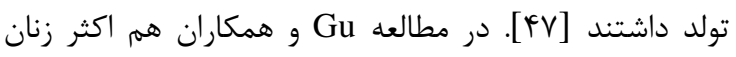

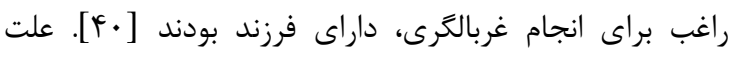

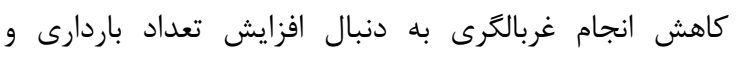

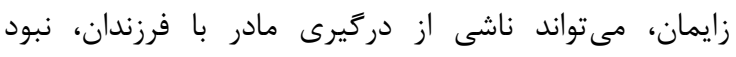

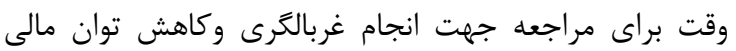

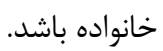

منظه براساس وضعيت تأهل تفاوت آمارى معنى دارى يافت نشد

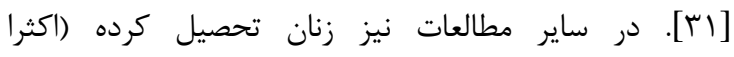

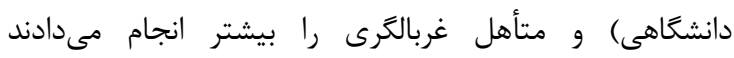

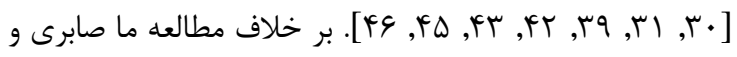

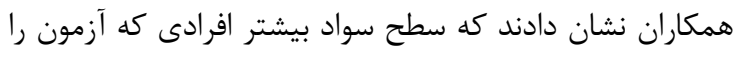

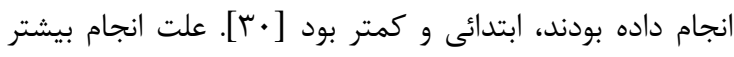

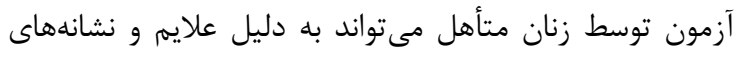

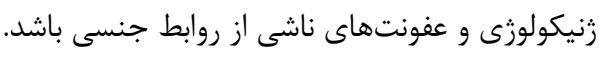

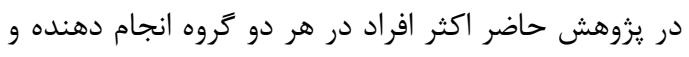

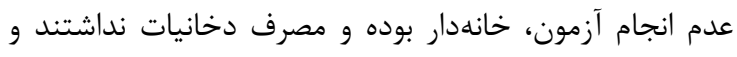

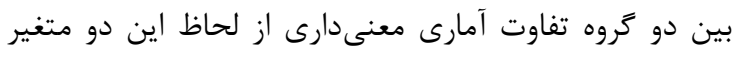

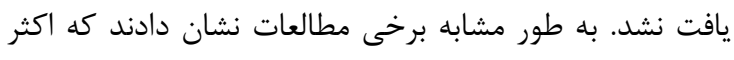

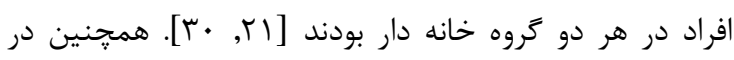

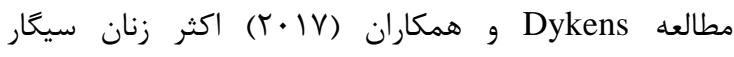

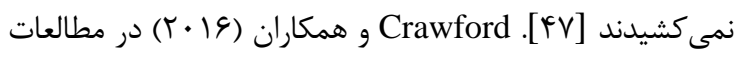

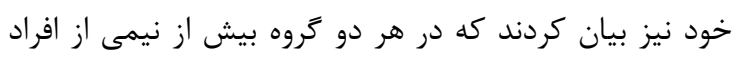

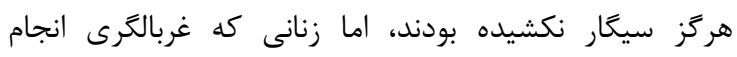

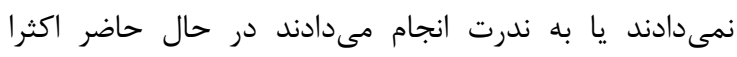

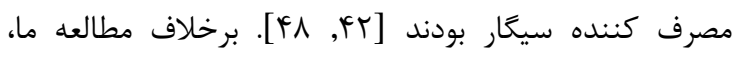
Mbachu

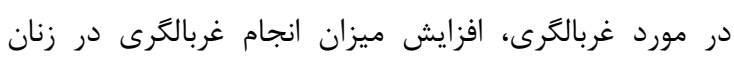

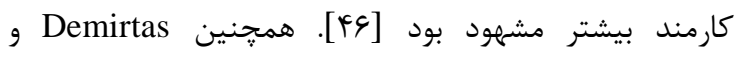

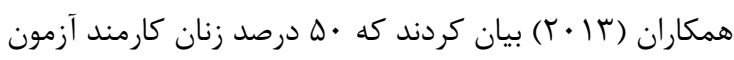

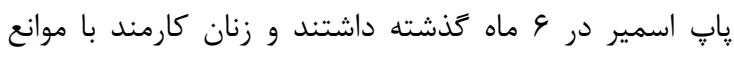

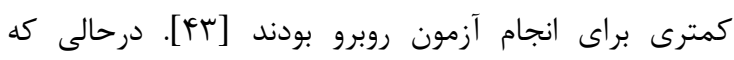

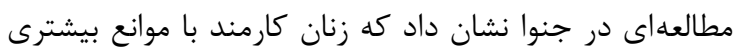

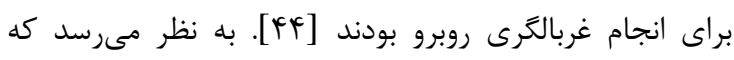

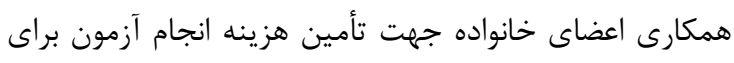

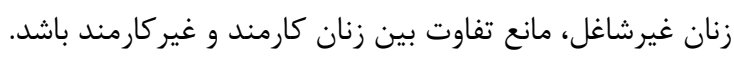

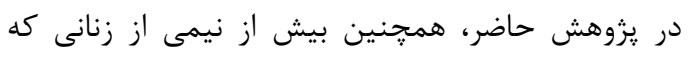

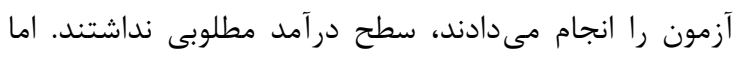

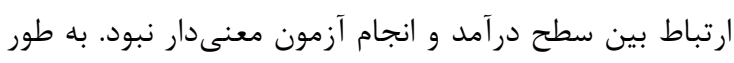

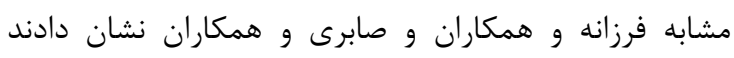

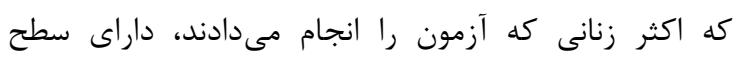

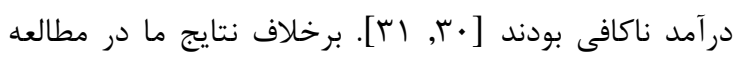

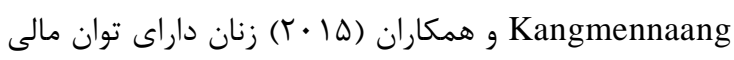

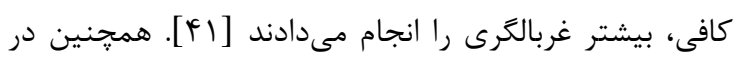

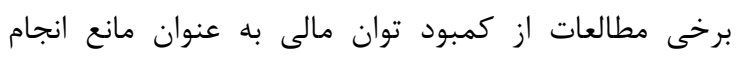

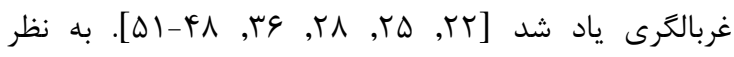

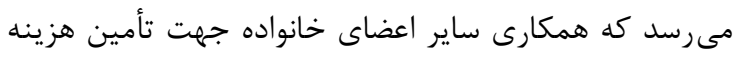

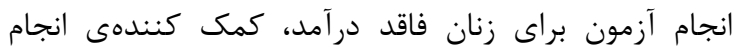
آزمون باشد.

در مطالعه حاضر بين انجام آزمون و سابقه خانوادكى سرطان

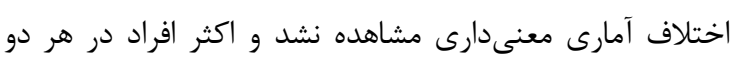




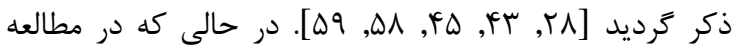
Mukama دربارهى سرطان دهانه رحم آكاهى داشتند [هان]. بنابراين

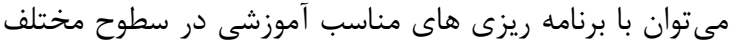

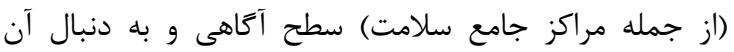

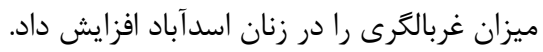

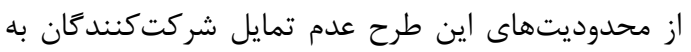

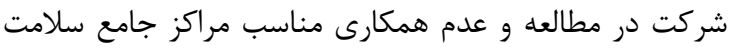

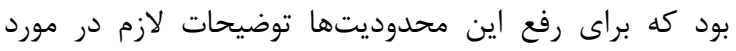

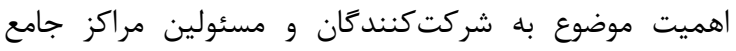

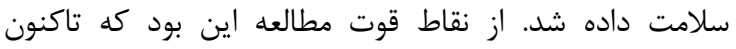
مطالعه اى در اين زمينه در شهر اسدآباد انجام نكرفته بود.

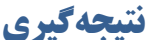

نتايج اين تحقيق، عملكرد پايين زنان شهر اسدآباد را در

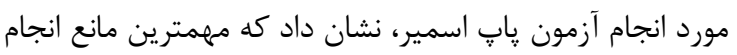

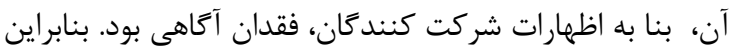

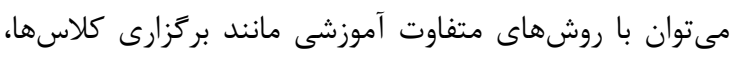

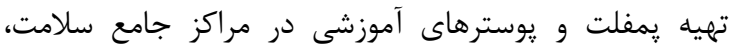

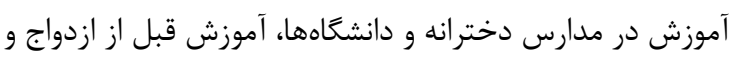

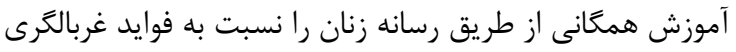

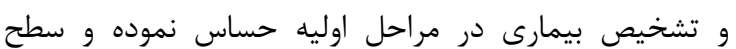
عملكرد را ارتقا بخشيد.

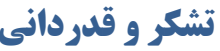

اين مقاله از طرح شماره تحقيقات و فناورى دانشعاه علوم يزشكى همدان استخراج

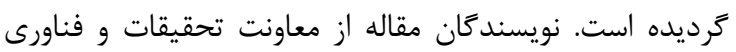
دانشعاه علوم يزشكى همدان به خاطر حمايت مالى اين مطالعه،

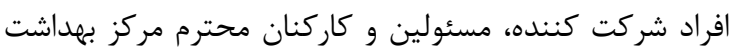
شهرستان اسدآباد كه در اين مطالعه همكارى نموده اند كمال تشكر و قدردانى را دارند.

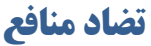
اين مطالعه براى نويسندًَان هيجَّونه تضاد منافعى نداشته

\section{REFERENCES}

1. Smeltzer SC, Gore BL, Hinkle JH, Cheever K. surgical nursing brunner svdas. 20ed. publishing community.2010.

2. Azizi F, Janghorbani M, Hatami H. Epidemiology and Control of common diseases in Iran. 3, editor. 2013.

3. Phiphs L. Nursing in cancer .Tehran: Chaher publishing: Translated by Sharoki A; 1994.

4. World Health Organization. Human Papillomavirus (HPV) and Cervical Cancer. Fact Sheet. . Accessed online 14th November 2016 at http://www whoint/mediacentre/ factsheets/fs380/en/. Updated June 2016.
يافتهها نشان داد اكثر زنانى كه آزمون را انجام مىدادند،

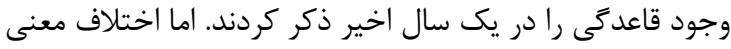

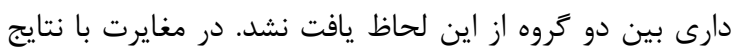

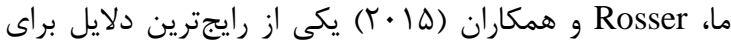

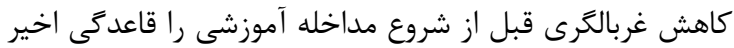

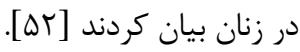

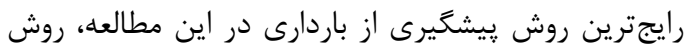

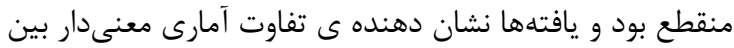

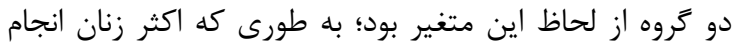

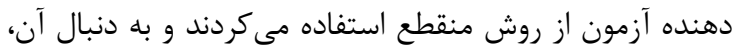

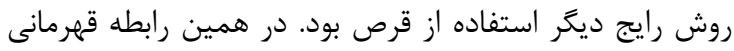
نسب و همكاران نشان دادند كه

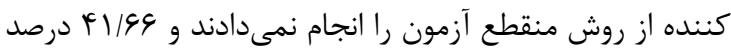

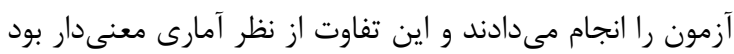

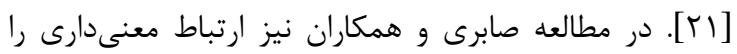

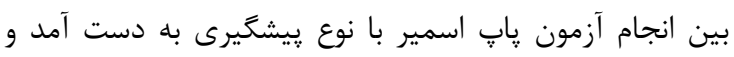

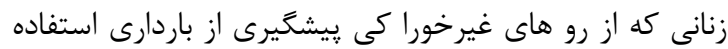

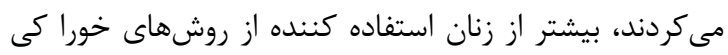

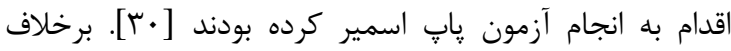

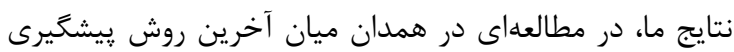

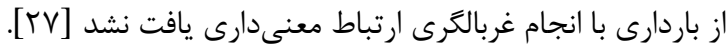

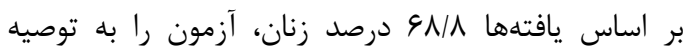
يزشك يا ماما انجام داده بودند. برخى نتايج همراستا با نتايج تحقيق حاضر بود [آ, צr,

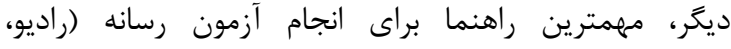

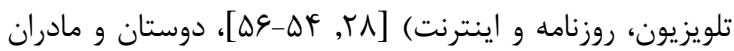

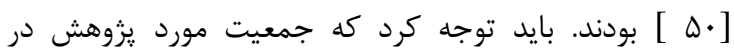

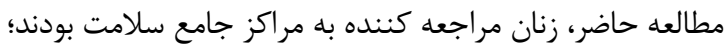

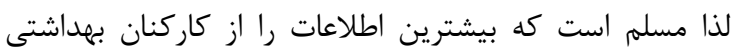
كسب خواهند كرد.

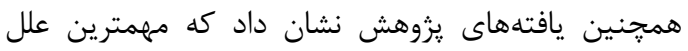

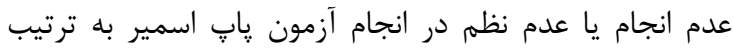

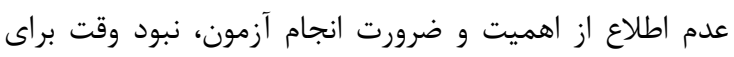

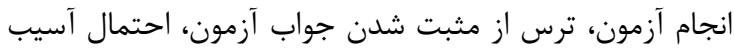

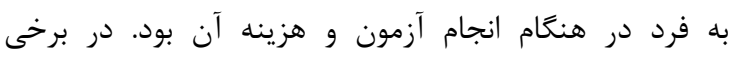

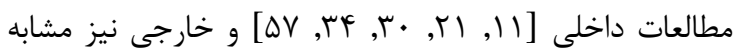
نتايج ما، عدم آكاهى دربارهى غربالكرى به اله عنوان مهمترين مانع

5. Khodakarami N, Farzane F, Yavari P, Khayyam Zade M, Taheri Panah R, Akbari MI. New cervical cancer screening program for low-risk women in Iran. Journal of Obstetrics Gynecology Iran. April 2014;17(95):8-17. (Persian)

6. World Health Organization. Information Centre on HPV and Cervical Cancer (HPV Information Centre): Summary Report on HPV and Cervical Cancer Statistics in South Africa. (Onlin), Available: http://www.who. Int/ hpvcentre. accessed 27/06/2013. 2007. 
7. Iran Ministry of Health.Country reports of cancer registries 2009.[Persian]. Available on :http://www.ircancer.ir/Portals /0/CancerBooks/Iran\%20Cancer\%20Report\%20. 1388.pdf. (2012).[Accessed 3 june 2016].

8. Black C. Working For a Healthier Tomorrow. Dome Carol Black's Review of the health of Britain's working age population. London: TSO. 2008.

9. Me Dohold SH. Performance and reporting of clinical breast examination:A review of literature cancer, Journal for clinicians, New York, Woll/ Pec; 54;2000, pp.345.

10. Program. Ncs. Breast srceening: the facts [Online]. [12 screen]. Available from: 2009 [cited 2009 Feb 10]:URL: http://www.cancerscreening.nhs.uk/breastscreen/publication s/nhsbsp-the-facts-english-2009.pdf.

11. Keshavarzian K, Barzegari Z. The Knowledge of Herisian Female about the Cervical Cancer and Pap Smear in 2012. Medical Journal of Tabriz University of Medical Sciences and Health Services. May. 2014;36(1):70-3. (Persian)

12. WHO. Comprehensive cervical cancer control-A guide to essential practice. Geneva: WHO Press.: 2014.

13. Farshbaf-Khalili A, Salehi-Pourmehr H, Shahnazi M, Yaghoubi S, Gahremani-Nasab P. [WHO guidelines for screening and treatment of precancerous lesions for cervical cancer prevention]. 2013. Available from: http://apps.who.int/iris/bitstream/10665/94830/5/978924154 8694 per.pdf. Persian. 2013

14. Farshbaf-Khalili A, Salehi-Pourmehr H, Shahnazi M, Yaghoubi S, Gahremani-Nasab P. Cervical cancer screening in women referred to healthcare centres in Tabriz, Iran. Niger Med J. 2015; 56(1):28-34. (Persian)

15. Solomon D, Breen N, McNeel T. Cervical cancer screening rates in the United States and the potential impact of implementation of screening guidelines CA. Cancer J Clin. 2007;57(2):105-11.

16. Allahverdipour H, Emami A. Perceptions of cervical cancer threat, benefits, and barriers of Papanicolaou smear screening programs for women in Iran. . Women Health. 2008:47(3):23-37. (Persian)

17. Krivak TC, Mcbroom JW, Elkas JC. Cervical and vaginal cancer. In: Berek JS. Novak's gynecology. 13th ed. 2002; Philadelphia: Lippincott Williams \& Wilkins; 1199-237.

18. Centers for Disease Control and Prevention. Gynecologic Cancers: Cervical cancer risk factors. Available from: http://www.cdc.gov/cancer/cervical/basic_info/risk_factors. htm. updated:May 6, 2014

19. Waller J, Bartoszek M, Marlow L, Wardle J. Barriers to cervical cancer screening attendance in England: a population-based survey. Journal of Medical Screening. 2009;16:199-204.

20. Wong LP, Wong YL, Low WY, Khoo EM, Shuib R. Cervical cancer screening attitudes and beliefs of Malaysian women who have never had a pap smear: a qualitative study. International Journal of Behavioral Medicine 2008;15: 289-92.

21. Ghahremany nasab P, Shahnazi M, Farshbaf Khalili A, Ghanbari S. Factors related to cervical cancer screening in women Referred to health centers in Tabriz 2011-2012. Journal of Obstetrics Gynecology Iran. March 2014;16(89):15-24. (Persian)

22. Refaei M, Dehghan Nayeri N, Khakbazan Z, Pakgohar M. Cervical Cancer Screening in Iranian Women: Healthcare Practitioner Perceptions and Views. Asian Pac J Cancer Prev. 2017;18(2):357-63

23. Akinlotan M, Bolin JN, Helduser J, Ojinnaka C, Lichorad A, McClellan D. Cervical Cancer Screening Barriers and Risk Factor Knowledge Among Uninsured Women. J Community Health. 2017:1-12.

24. Dhivya B, Balakrishnan PR. Cervical cancer screening: Knowledge, attitude and practices in a primary health centre in rural India. 2015;24(35):36-50.

25. Parsa P, Sharifi F, Shobeiri F, Karami M. The effect of group counseling on cervical cancer screening knowledge and practice among rural women in kaboudarahang city, hamadan. The J Urmia Nurs Midwifery Fac. 2017; 14(10):14-25.

26. Bahmani A, Rahmani K, Ahmadian F, Alizadeh Z, Akhtar
B. Explanation of Pap Smear Preventive Behavior among Women Based on Health Belief Model: A Qualitative Study. Iran J Health Educ Health Promot. 2017;5(1):Abstract.

27. Bakht R, Shobairi F, Roshanaei GH, Fakori E. Factors Affecting the Screening of Cervical Cancer in Addicted Women in Hamadan in 2017: Based on the Model of Protection Motivation Theory. Hakim Seyed Esmail Jorjani Jour. 2017.(2)4. (Persian)

28. Ndejjo R, Mukama T, Kiguli J, Musoke D. Knowledge, facilitators and barriers to cervical cancer screening among women in Uganda: a qualitative study. BMJ Open. 2017;7:1-9.

29. Tork Zahrani S, Rastegari L, Khoda Karami N, Mohebbi P. Relationship between Quality of Life and Social Support in Women Treated for Cervical Cancer. Journal (NMCJ) Nursing and Midwifery Care. 2012;1(2):51 -7. (Persian)

30. Saberi F, Sadat Z, Abedzadeh Kalahrod M. Factors associated with cervical cancer screening (cervical) and its barriers for women. Institute of Health Sciences. 2011; 11(3):365-70. (Persian)

31. Farzaneh E, Heydari H, Shekarchi A, Kamran A. Breast and cervical cancer-screening uptake among females in Ardabil, northwest Iran: a community-based study. OncoTargets and Therapy. 2017;10:985-92.

32. Ezzati E, Shriat F, Moradi F, Babazadeh T. The Effect of a Blended Educational Program (BEP) on Cervical Cancer Screening Behavior among Housekeeper Women in West Eslamabad in 2016:an Application of Health Belief Model. Scientific Journal of Ilam University of Medical Sciences. 2016;25(1):110-20.

33. Hajializadeh K, Ahadi H, Jomhari F, Rahgozar M. The Role of Health Beliefs in Predicting Barriers to Cervical Cancer Screening. Journal of Kerman University of Medical Sciences. 2014;21(5):416-25. (Persian)

34. Mohebi S, Sharifirad G, Gharlipour Z, Kamran A. The Study of Pap Smear Conduction and its Related Factors Based on Health Belief Model in Women Referring to Health Care Centers in Qom During year 2014. J Educ Community Health. 2016;2(4):25-33.

35. Ruzigana G, Bazzet-Matabele L, Rulisa S, Martin AN, Ghebre RG. Cervical cancer screening at a tertiary care center in Rwanda. Gynecol Oncol Rep. 2017;21:13-6.

36. Assoumou SZ, Mabika Mabika B, Mbiguino AN, Mouallif M, Khattabi A, Ennaji M. Awareness and knowledge regarding of cervical cancer, Pap smear screening and human papillomavirus infection in Gabonese women. BMC Women's Health. 2015;15(37):1-7.

37. Morowatisharifabad M, Norouzi S, Layeghy T, Norouzi A. Survey of Cervix Cancer Screening Determinants among 20-65 Years Old Women Based On Health Belief Model in Lordegan, Chahar Mahal Bakhtiyaree, 2009 .Yazd. 2013;12(1):98-106.

38. Chorley AJ, Marlow LAV, Forster AS, Haddrell JB, Waller $\mathrm{J}$. Experiences of cervical screening and barriers to participation in the context of an organised programme: a systematic review and thematic synthesis. PsychoOncology, 2017; 72; 161.

39. Kasting ML, Wilson S, Zollinger TW, Dixon BE, Stupiansky NW, Zimet GD. Differences in cervical cancer screening knowledge, practices, and beliefs: An examination of survey responses. Preventive Medicine Reports. 2017;5:169-74.

40. Gu C, Chan CWH, He GP, Choi KC, Yang SB. Chinese women's motivation to receive future screening: The role of social-demographic factors, knowledge and risk perception of cervical cancer. European Journal of Oncology Nursing. 2013;17:154-61.

41. Kangmennaang J, Thogarapalli N, Mkandawire P, Luginaah I. Investigating the disparities in cervical cancer screening among Namibian women. Gynecologic Oncology 2015; 138:411-6.

42. Crawford A, Benard V, King J, Thomas CC. Understanding Barriers to Cervical CancerScreening in Women With Access to Care, Behavioral Risk Factor Surveillance System, 2014. Prev Chronic Dis. 2016;13.

43. Demirtas B, Acikgoz I. Promoting Attendance at Cervical 
Cancer Screening: Understanding the Relationship with Turkish Womens' Health Beliefs. Asian Pacific J Cancer Prev. 2013;14(1):333-40.

44. Catarino RR, Vassilakos PP, Royannez-Drevard II, Guillot CC, Alzuphar SS, Fehlmann AA, et al. Barriers to Cervical Cancer Screening in Geneva (DEPIST Study). J Low Genit Tract Dis. 2016;20(2):135-8.

45. Compaore S, Ouedraogo CM, Koanda S, Haynatzki G, Chamberlain RM, Soliman AS. Barriers to Cervical Cancer Screening in Burkina Faso: Needs for Patient and Professional Education. J Cancer Educ. 2016;31(4):760-6.

46. Mbachu C, Dim C, Ezeoke U. Effects of peer health education on perception and practice of screening forcervical cancer among urban residential women in southeast Nigeria: a before and after study. BMC women's health. 2017;17(1):41

47. Dykens JA, Linn AM, Irwin T, Peters KE, Pyra M, Traoré $\mathrm{F}$, et al. Implementing visual cervical cancer screening in Senegal: a cross-sectional study of risk factors and prevalence highlighting service utilization barriers. International Journal of Women's Health. 2017;9:59-67.

48. Crawford J, Ahmad F, Beaton D, Bierman AS. Cancer screening behaviours among South Asian immigrants in the UK, US and Canada: a scoping study. Health and Social Care in the Community. 2016;24(2):123-53.

49. Hope KA, Moss E, Redman CWE, Sherman SM. Psychosocial influences upon older women's decision to attend cervical screening: A review of current evidence. Preventive Medicine 2017;101:60-6.

50. Increasing Cervical Cancer Screening in Underserved Populations. JCN. 2017; 34( 3):49-50.

51. Buchanan Lunsford N, Ragan K, Lee Smith J, Saraiya M, Aketch M. Environmental and Psychosocial Barriers to and Benefits of Cervical CancerScreening in Kenya. (C) AlphaMed Press 2017. 2017;22(2):173-81.
52. Rosser JI, Njoroge B, Huchko MJ. Changing knowledge, attitudes, and behaviors regarding cervical cancer screening: The effects of an educational intervention in rural Kenya. Patient Educ Couns. 2015 98(7):884-9.

53. Karimy M, Gallali M, Niknami S, Aminshokravi F, Tavafian SS. The effect of health education program based on Health Belief Model on the performance of Pap smear test among women referring to health care centers in Zarandieh. Journal of Jahrom University of Medical Sciences. 2012;10(1):53-9.

54. Almobarak AO, Elbadawi AA, Elmadhoun WM, Elhoweris MH, Ahmed MH. Knowledge, Attitudes and Practices of Sudanese Women Regarding the Pap Smear Test and Cervical Cancer. Asian Pac J Cancer Prev. 2016;17(2): 625-30.

55. Mukama T, Ndejjo R, Musabyimana A, Halage AA, Musoke D. Women's knowledge and attitudes towards cervical cancer prevention: a cross sectional study in Eastern Uganda. BMC Women's Health. 2017;17(9):1-8.

56. Isa Modibbo F, Dareng E, Bamisaye $P$, Jedy-Agba E, Adewole A, Oyeneyin L, et al. Qualitative study of barriers to cervical cancer screening among Nigerian women. BMJ. 2016;6:1-12.

57. Keshavarz Z, Simbar M, Ramazankhani A, Alavimajd H. 2011;9(3):23-36

58. Abiodun OA, Olu-Abiodun OO, Sotunsa JO, Oluwole FA. Impact of health education intervention on knowledge and perception of cervical cancer and cervical screening uptake among adult women in rural communities in Nigeria. BMC Public Health. 2014; 14(814):1-9.

59. Di JL, Rutherford S, Wu JL, Song B, Ma L, Chen JY, et al. Knowledge of Cervical Cancer Screening among Health Care Workers Providing Services Across Different Socioeconomic Regions of China. Asian Pac J Cancer Prev. 2016;17(6):2965-72. 